\title{
Fast inverse approach for the deconvolution of ultrasonic TFM images using a spatially varying PSF in NDT
}

\author{
Nans Laroche ${ }^{1,2}$, Sébastien Bourguignon ${ }^{2}$, Ewen Carcreff ${ }^{1}$, Jérôme Idier $^{2}$ and Aroune Duclos ${ }^{3}$ \\ ${ }^{1}$ The Phased Array Company \\ Nantes, France \\ nans.laroche@tpac-ndt.com \\ ${ }^{2}$ Ecole Centrale de Nantes / \\ Laboratoire des Sciences \\ du Numérique de Nantes \\ Nantes, France \\ ${ }^{3}$ Laboratoire d'Acoustique \\ de l'Université du Maine \\ Le Mans, France
}

\begin{abstract}
Multi-element probes are widely used in NonDestructive Testing (NDT) for their ability to produce images. Full Matrix Capture (FMC) is a standard acquisition process that consists in acquiring all inter-element responses. The common reconstruction procedure, namely the Total Focusing Method (TFM), is a linear reconstruction technique that does not account for the instrumental response of the transducers. Thus, its resolution remains limited. The inversion of the direct model considering the acoustic response of the transducers and formulated on the FMC data is a heavy procedure in terms of computing resources and storage. In order to reduce the size of the problem, the proposed approach considers the beamformed TFM image as a back-projection of the data in the space domain. The proposed model linearly links the TFM image and the reflectivity map of the media under inspection. The PSF (Point Spread Function) associated to a pixel is the TFM reconstruction from data acquired on a reflectivity map where only this pixel is reflecting the signal. This raises a 2D "deconvolution" problem where the PSF is varying spatially. To limit the number of computations, the proposed method assumes that the variation of the PSF is slow, so that it can be interpolated accurately. The interpolation model involves convolution products that can be quickly computed using Fast Fourier Transforms (FFTs). The reconstructed image is obtained within an iterative procedure enforcing sparsity and spatial smoothness. The resolution improvement is evidenced from experimental data acquired from a stainless steel block containing Side Drilled Holes (SDH) inspected with a 128-element probe.
\end{abstract}

Index Terms-Non Destructive Testing, Total Focusing Method, Deconvolution, Point Spread Function

\section{INTRODUCTION}

The use of phased array probes has become very common in NDT [1] and medical imaging. Delay-and-Sum (DAS) methods such as the Total Focusing Method (TFM) [2] are used in real time for industrial applications thanks to the capabilities of Graphic Processing Units (GPU). Such postprocessing methods focus on each pixel of an image by simply summing all signals at the corresponding times of flight. Thus, the narrow bandwidth of the ultrasonic transducers limits the resolution of these methods to the Rayleigh criterion [3]. Some

The authors would like to thank the ANRT for partial funding (2017/1083). methods based on a sparse prior have been proposed in order to improve the resolution of ultrasonic images [4], [5]. However, the acoustic response of the transducers [6] is not considered in these models whose resolution hence remains limited. The acoustic response has been considered in an inverse approach proposed in [7] that computes highly resolved images from FMC data. Nevertheless, the computational cost of such a method is high due to the large size of the FMC data.

The approach proposed in this paper considers the beamformed TFM image as a back-projection of the ultrasonic data in the image domain. The Point Spread Function (PSF) associated to each pixel is the TFM image corresponding to a reflectivity map where only the considered pixel reflects the signals. This model formulates a 2D "deconvolution" problem with a spatially varying PSF. The computation of all PSFs being computationally expensive, we propose to interpolate the PSF at each pixel from the computation of a few reference PSFs. Then, a penalized least-squares criterion is minimized within an iterative procedure imposing sparsity and spatial continuity. This procedure can be fastly implemented for two main reasons. Firstly, the size of the optimization problem is independent of the size of FMC data. Secondly, matrix operations are convolution products that can be computed efficiently using Fast Fourier Transforms (FFTs). A variant of the interpolation model has been proposed in the context of Plane Wave Imaging (PWI) in the medical field [8] but appears hardly applicable in the context of FMC.

The paper is organized as follows. Section II presents the FMC acquisition process and the model that links the reflectivity image to the ultrasonic data. Section III presents the contribution of the paper at different stages: the deconvolution and interpolation models and the inversion scheme with sparsity and spatial smoothness priors. Section IV presents comparisons of computation times on synthetic data. Section V presents an experimental application on a tightly spaced set of flaws from a stainless steel block. 


\section{MODEL ON FMC DATA}

FMC acquisition consists in acquiring the signals from each emitter-receiver pair of a multi-element probe. For a probe with $N_{\mathrm{el}}$ transducers, $N_{\mathrm{el}}^{2}$ signals are recorded. The signal from the $i$-th emitter to the $j$-th receiver is denoted $y_{i j}(t)$. Each signal can be modeled as a convolution between the reflectivity of the media and an elementary waveform, defined as the acoustic response of the transducers distorted by the media [9]. Under this assumption, a waveform matrix $\mathbf{H}_{t}$ can be built to link the FMC data to the reflectivity image [7]:

$$
\boldsymbol{y}=\mathbf{H}_{t} \boldsymbol{o}+\boldsymbol{n}_{t},
$$

where $\boldsymbol{y} \in \mathbb{R}^{N_{t} N_{\mathrm{el}}^{2}}$ collects all the signals $y_{i j}, \boldsymbol{o} \in \mathbb{R}^{N_{x} N_{z}}$ represents the ultrasonic reflectivity and each column of $\mathbf{H}_{t} \in$ $\mathbb{R}^{N_{t} N_{\mathrm{el}}^{2} \times N_{x} N_{z}}$ gathers the signature in the data of a scatterer at the proper position in the reflectivity image. The term $\boldsymbol{n}_{t} \in$ $\mathbb{R}^{N_{t} N_{\mathrm{el}}^{2}}$ represents the noise and model errors.

The Total Focusing Method (TFM) [2] is a common method to process such data based on the computation of times of flight $\tau(x, z, i, j)$ which reads, in the case of an inspection in contact of a flat specimen:

$$
\tau(x, z, i, j)=\frac{\sqrt{\left(x-u_{i}\right)^{2}+z^{2}}+\sqrt{\left(x-v_{j}\right)^{2}+z^{2}}}{c},
$$

where $u_{i}$ and $v_{j}$ respectively denote the abscissa of the emitter and receiver and $c$ is the sound velocity. The principle of TFM consists in summing all the signals at the proper times of flight for each pixel $(x, z)$ :

$$
o_{\mathrm{TFM}}(x, z)=\sum_{i=1}^{N_{\mathrm{el}}} \sum_{j=1}^{N_{\mathrm{el}}} y_{i, j}(\tau) \Longleftrightarrow \boldsymbol{o}_{\mathrm{TFM}}=\mathbf{B} \boldsymbol{y} .
$$

That is, the TFM can be described (up to discretization errors) as the application of a binary matrix $\mathbf{B}$ to the data $\boldsymbol{y}$, where each column of B contains only one "1" at the corresponding time of flight.

\section{DIRECT MODEL ON TFM IMAGE AND INVERSION}

The reflectivity map $\boldsymbol{o}$ contains high frequencies that have been filtered out by the observation process due to the limited bandwidth of the transducers. Thus, the inverse problem defined in (1) is ill-posed. The inversion of such a model (noted Inversion T) has been performed by considering a sparse $a$ priori in [7] to reconstruct highly resolved ultrasonic images. The reconstructed image is the solution of the optimization problem:

$$
\boldsymbol{o}_{t}=\underset{\boldsymbol{o}}{\arg \min }\left\|\boldsymbol{y}-\mathbf{H}_{t} \boldsymbol{o}\right\|^{2}+\phi(\boldsymbol{o}) .
$$

where $\phi$ is a penalization function. The regularization is performed using sparsity and spatial smoothness priors:

$$
\phi(\boldsymbol{o})=\mu_{1}\|\boldsymbol{o}\|_{1}+\mu_{2}\|\mathbf{D o}\|^{2},
$$

where $\mathbf{D}$ is a matrix computing differences between neighbor pixels. This inversion requires a lot of computations due to the large size of FMC data.
We propose to reduce the number of computations by combining (1) and (3) in order to link the TFM image to the reflectivity linearly:

$$
\boldsymbol{o}_{\mathrm{TFM}}=\mathbf{H}_{s} \boldsymbol{o}+\boldsymbol{n}_{s}, \text { with } \mathbf{H}_{s}=\mathbf{B H}_{t} .
$$

This model formulates another inverse problem in the image domain. The TFM image is considered as a spatial projection of the data on the reconstruction grid. Each column of the matrix $\mathbf{H}_{s}$ represents the PSF associated to a pixel, that is, the TFM image corresponding to the reflectivity map where only this pixel reflects the signal. We consider that the loss of information resulting from this projection can be compensated by accurately modeling the PSF in the matrix $\mathbf{H}_{s}$. In practice, the computation of the PSF associated to each pixel requires a lot of computational and storage resources. Besides, it is natural to consider that the spatial variations of the PSF are slow and that the PSF can be interpolated accurately from a few PSFs spread across the grid [10]-[12]. The PSF spatial variation is emphasized in Fig. 1. Fig. 1 (a) represents 25 PSFs spread across a grid corresponding to a 64-element probe pulsing in a medium where the wavelength is equal to $1 \mathrm{~mm}$ and the inter-element space is $0.5 \mathrm{~mm}$. Fig. 1 (b), (c) and (d) are focused views on three different PSFs. The slow spatial variations are clearly visible on these figures, as well as the interest to interpolate them. Notation $\boldsymbol{r}=(x, z)$ represents the
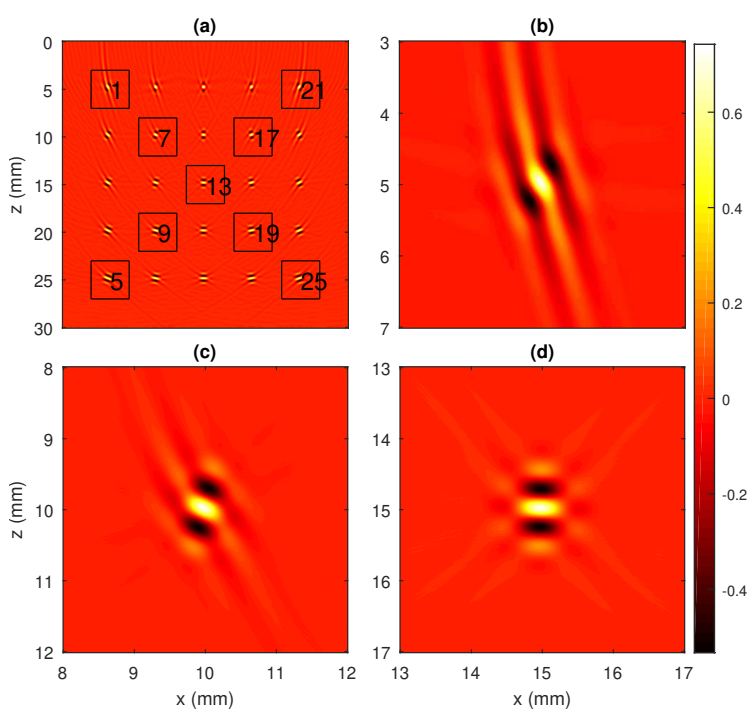

Fig. 1. Example of PSFs selected at several places in the reconstruction grid. Fig. 1 (a) represents the sum of 25 PSFs spread over the image. Fig. 1 (b), (c) and (d) respectively focus on PSF 1,7 and 13.

spatial coordinate of a pixel and $h_{\boldsymbol{r}}$ is the associated PSF. From the computation of a limited number $N_{\mathrm{PSF}}$ of PSFs $h_{i}$ located at pixels $\boldsymbol{r}_{i}=\left(x_{i}, z_{i}\right), i=1, \ldots, N_{\mathrm{PSF}}$, the PSF at pixel $\boldsymbol{r}_{0}$ can be approximated as:

$$
h_{\boldsymbol{r}_{0}}(\boldsymbol{r}) \approx \sum_{i=1}^{N_{\mathrm{PSF}}} w_{i}\left(\boldsymbol{r}_{0}\right) h_{i}(\boldsymbol{r})
$$

where $w_{i}$ are weighting coefficients depending on the distance between the pixel of interest $\boldsymbol{r}_{0}$ and the pixel $\boldsymbol{r}_{i}$. In the 
proposed method, the Inverse Distance Weighting (IDW) [10] is used to compute these coefficients:

$\forall j=1, \ldots, N_{\mathrm{PSF}} ; w_{i}\left(\boldsymbol{r}_{j}\right)=1$ if $i=j$ and 0 otherwise

and $w_{i}\left(\boldsymbol{r}_{0}\right)=\left\|\boldsymbol{r}_{0}-\boldsymbol{r}_{i}\right\|^{-1} / \sum_{j=1}^{N_{\mathrm{PSF}}}\left\|\boldsymbol{r}_{0}-\boldsymbol{r}_{j}\right\|^{-1}$ elsewhere.

The term corresponding to the pixel $\boldsymbol{r}_{0}$ in the product $\mathbf{H}_{s} \boldsymbol{o}$ can than be computed as follows:

$\left(\mathbf{H}_{s} \boldsymbol{o}\right)\left(\boldsymbol{r}_{0}\right)=\sum_{\boldsymbol{r}} h_{\boldsymbol{r}}\left(\boldsymbol{r}_{0}-\boldsymbol{r}\right) o(\boldsymbol{r}) \approx \sum_{\boldsymbol{r}} \sum_{i=1}^{N_{\mathrm{PSF}}} w_{i}(\boldsymbol{r}) h_{i}\left(\boldsymbol{r}_{0}-\boldsymbol{r}\right) o(\boldsymbol{r})$,

which can be reformulated using convolution products:

$$
\left(\mathbf{H}_{s} \boldsymbol{o}\right)\left(\boldsymbol{r}_{0}\right) \approx \sum_{i=1}^{N_{\mathrm{PSF}}}\left(h_{i} * o_{i}\right)\left(\boldsymbol{r}_{0}\right),
$$

with $o_{i}(\boldsymbol{r})=w_{i}(\boldsymbol{r}) o(\boldsymbol{r})$. Thus the matrix products involving $\mathbf{H}_{s}$ can be approximated as:

$$
\mathbf{H}_{s} \boldsymbol{o} \approx \sum_{i=1}^{N_{\mathrm{PSF}}} \mathbf{H}_{i}\left(\mathbf{W}_{i} \boldsymbol{o}\right), \text { and } \mathbf{H}_{s}^{t} \boldsymbol{o} \approx \sum_{i=1}^{N_{\mathrm{PSF}}} \mathbf{W}_{i}\left(\mathbf{H}_{i}^{t} \boldsymbol{o}\right),
$$

with $\mathbf{H}_{i}$ the discrete convolution matrix associated to the PSF $h_{i}$ and $\mathbf{W}_{i}$ the diagonal matrix containing the weights $w_{i}$. The computational cost of these products is $N_{\mathrm{PSF}}$ convolutions, computed quickly using FFTs. These operations yield the solution (noted Inversion S) of the optimization problem:

$$
\boldsymbol{o}_{s}=\underset{\boldsymbol{o}}{\arg \min }\left\|\boldsymbol{o}_{\mathrm{TFM}}-\mathbf{H}_{s} \boldsymbol{o}\right\|^{2}+\phi(\boldsymbol{o}),
$$

Optimization is performed with FISTA. The iterative procedure is implemented in Matlab once the TFM image and the PSF are computed.

\section{COMPARISON OF COMPUTATION TIMES ON SYNTHETIC DATA}

Computation times have been compared in the configuration used in Fig. 1. A pair of close flaws distant from $\lambda / 2$ is placed at the center of each of the 25 areas. Three methods have been compared : Inversion T detailed in [7] computed with Matlab and GPU and Inversion S with 1 (area 13) and 4 PSFs (areas 7 , $9,17,19)$ computed with Matlab. Results are shown in Fig. 2. The upper graph shows the influence of the number of pixels in the reconstructed image with a fixed number of transducers equal to 64 . For each method, the computation time increases with the number of pixels but it remains at least 10 times smaller for Inversion S. The lower graph shows the influence of the transducer number with a fixed number of pixels equal to $251^{2}$. Note that the computing time of the proposed method is nearly independent of the number of transducers. Indeed, this value only influences the pre-computation time of TFM and PSF, which is negligible.
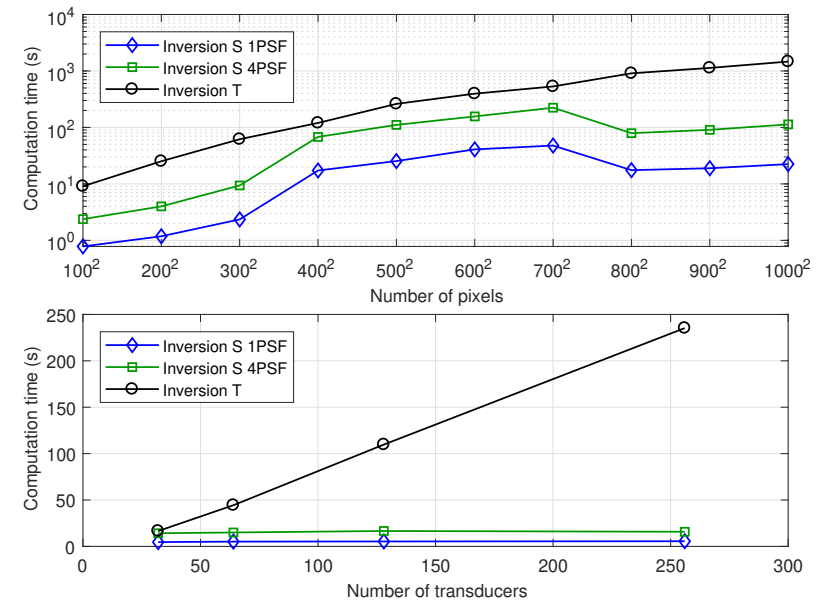

Fig. 2. Computation times of Inversion $\mathrm{T}$ and Inversion $\mathrm{S}$ with 1 and 4 PSFs.

\section{APPLICATION TO RESOLUTION ENHANCEMENT OF TFM IMAGES}

The purpose of this experiment is to emphasize both the resolution capability of the proposed method and the benefit of the PSF interpolation model. Data have been acquired using a 128-element probe designed by Imasonic (Voray-sur-l'Ognon, France), pulsing around $10 \mathrm{MHz}$, with an inter-element space equal to $0.3 \mathrm{~mm}$. The experimental test block is made of Stainless Steel $304(c=5650 \mathrm{~m} / \mathrm{s})$ and contains close side drilled holes of $0.3 \mathrm{~mm}$ diameter at $50.8 \mathrm{~mm}$ depth. On this experiment, the Rayleigh criterion is equal to $0.98 \mathrm{~mm}$. The smallest distance between two close flaws is theoretically around $0.4 \mathrm{~mm}$ center to center, i.e. $0.1 \mathrm{~mm}$ edge to edge. In practice, the distance between flaws is not well known and Inversion $\mathrm{T}$ has been considered as a reference. Fig. 3 (a) is a picture of the experiment and Fig. 3 (b) is a TFM reconstruction on a large view showing the position of the reconstruction grid and the transducers. The proposed method has been tested
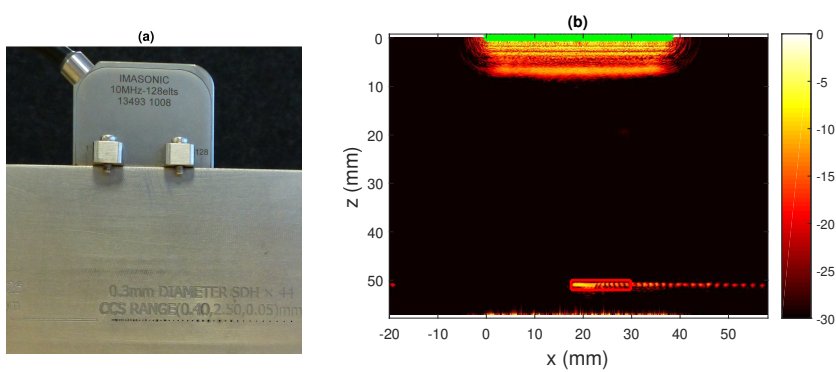

Fig. 3. (a) Picture of the inspection. (b) TFM reconstruction, transducers are marked with a green $\operatorname{star}(*)$ and the reconstruction grid is framed in red.

with one and three PSFs represented in Fig. 4 (a), the central one being used for the deconvolution with one PSF. The two others are estimated at $2 \mathrm{~mm}$ from the extremities of the image but not at the edge to avoid side effects. The elementary waveform used to compute the PSF is a Gaussian echo. The center frequency, bandwidth and phase of the Gaussian echo are estimated on the backwall echo positioned at $76.2 \mathrm{~mm}$ 
by minimizing a non linear least-squares criterion using the Levenberg-Marquardt algorithm. Reconstructions are shown in Fig. 4. The proposed inverse method using one PSF improves significantly the resolution of the image compared to the TFM but is not able to separate the closest flaws around $x=19 \mathrm{~mm}$. These flaws are well separated in the reconstruction using three PSFs which emphasizes the relevance of the proposed method. Fig. 5 shows the mean of pixel intensities over the $\mathrm{z}$ direction

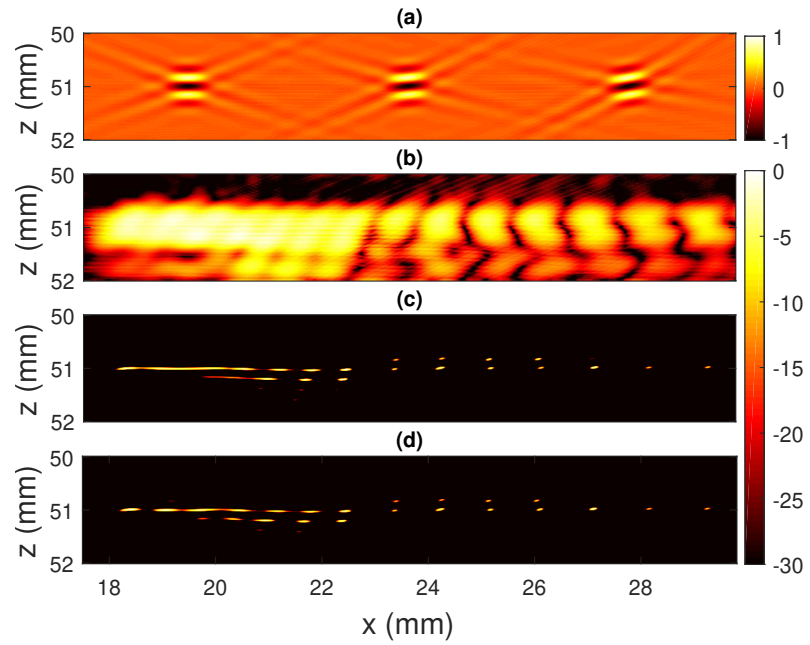

Fig. 4. Image of the sum of the three PSFs used for the inversion (a). TFM reconstruction (b), Inversion S 1 PSF (c) and 3 PSFs (d).

from previous reconstructions. The black diamonds indicate the position of the flaws computed from the Inversion $\mathrm{T}$. The upper figure clearly shows the benefit of the proposed method to improve the resolution compared to TFM. The lower figure is a zoom on the closest flaws showing the benefit of using the interpolation method. In this experiment, the reconstructed grid has $201 \times 1231$ pixels and the reconstruction time of TFM and Inversion $\mathrm{S}$ with one and three PSFs are $0.33 \mathrm{~s}, 7.57 \mathrm{~s}$ and 25.87 s respectively. On this data set involving a large number of transducers and pixels, the computation time of the Inversion $\mathrm{T}$ chosen as reference is around $450 \mathrm{~s}$.

\section{CONCLUSIONS}

This work proposes an inverse method to improve the resolution of ultrasonic images from FMC data. Whereas [7] performed the inversion from the large size FMC data, we propose to compute the back-projection of the FMC data in the space domain first, and then to perform the inversion step from the resulting smaller size TFM image. Finding the exact solution of this problem implies the computation of the PSF at each pixel of the grid, which has a heavy computational cost. The proposed interpolation method limits these computations while keeping a good precision of reconstruction. It also enables the fast computation of matrix products using FFTs. Therefore, this method is able to reconstruct high resolution images with a reasonable computation time and can be considered for industrial applications.

A wiser choice of the number and positions of the PSFs is a potential lever to improve the quality of the reconstructed

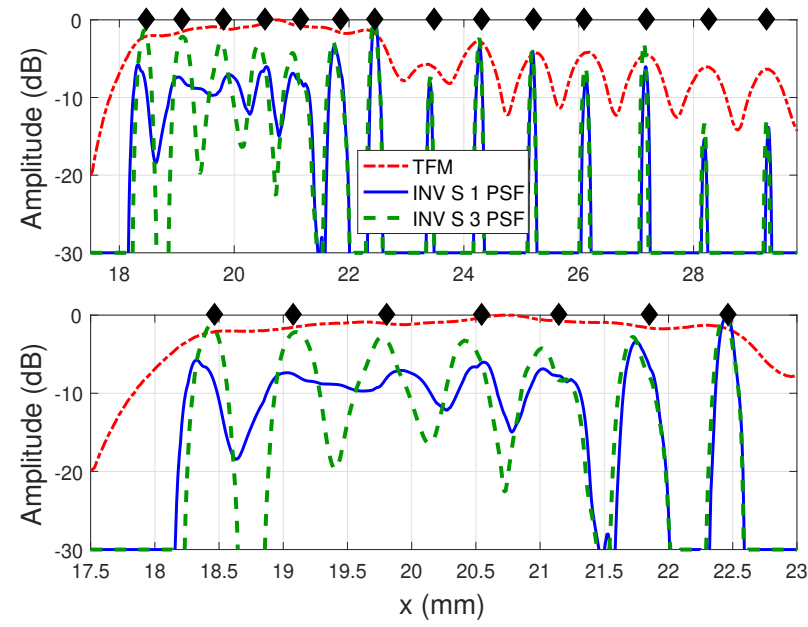

Fig. 5. Intensity curves (in $\mathrm{dB}$ ) of reconstructed reflectivity around the flaws depth for TFM (.-), Inversion S 1 PSF (-) and 3 PSFs(--).

image. This method could also be applied to complex materials by coupling the spatial variations of the PSF to acoustic models. Such a method could be relevant in order to separate close flaws in scattering materials.

\section{ACKNOWLEDGMENT}

We would like to thank George D. Connolly from Electric Power Research Institute (EPRI, Charlotte, USA) who lent us the experimental specimen.

\section{REFERENCES}

[1] J. Krautkramer and H. Krautkramer, Ultrasonic Testing of materials. Berlin: Springer-Verlag, 1990.

[2] C. Holmes, B. Drinkwater, and P. Wilcox, "Post-processing of the full matrix of ultrasonic transmit-receive array data for non-destructive evaluation," NDT\&E INT, vol. 38, no. 8, pp. 701-711, Dec. 2005.

[3] F. Simonetti, "Multiple scattering: The key to unravel the subwavelength world from the far-field pattern of a scattered wave," Phys. Rev. E, vol. 73, no. 3, Mar. 2006.

[4] T. Szasz, A. Basarab, and D. Kouame, "Beamforming Through Regularized Inverse Problems in Ultrasound Medical Imaging," IEEE Trans. Ultrason., Ferroelectr., Freq. Control, vol. 63, no. 12, pp. 2031-2044, Dec. 2016.

[5] E. Ozkan, V. Vishnevsky, and O. Goksel, "Inverse Problem of Ultrasound Beamforming With Sparsity Constraints and Regularization," IEEE Trans. Ultrason., Ferroelectr., Freq. Control, vol. 65, no. 3, pp. 356365, Mar. 2018.

[6] J. Jensen, "Estimation of Pulses in Ultrasound B-Scan Images," IEEE Trans. Med. Imag., vol. 10, no. 10, pp. 164-172, 1991.

[7] N. Laroche, E. Carcreff, S. Bourguignon, J. Idier, and A. Duclos, "An inverse approach for ultrasonic imaging by total focusing point for close reflectors separation," in 2018 IEEE Int. Ultrason. Symp., Oct 2018.

[8] A. Besson, L. Roquette, D. Perdios, M. Simeoni, M. Arditi, P. Hurley, Y. Wiaux, and J.-P. Thiran, "A Physical Model of Non-stationary Blur in Ultrasound Imaging," IEEE Trans. Comput. Imaging, pp. 1-1, 2019.

[9] K. V. Gurumurthy and R. Martin Arthur, "A dispersive model for the propagation of ultrasound in soft tissue." Ultrasonic Imaging, vol. 4, pp. 355-377, 1982

[10] M. Gentile, F. Courbin, and G. Meylan, "Interpolating point spread function anisotropy," Astron. Astrophys., vol. 549, p. A1, Jan. 2013.

[11] L. Denis, E. Thiébaut, F. Soulez, J.-M. Becker, and R. Mourya, "Fast Approximations of Shift-Variant Blur,” Int. J. Comput. Vis., vol. 115, no. 3, pp. 253-278, Dec. 2015.

[12] L. Roquette, M. Simeoni, P. Hurley, and A. Besson, "On an analytical, spatially-varying, point-spread-function," in 2017 IEEE Int. Ultrason. Symp., Sep. 2017. 